\title{
Pathogenesis of Meningococcemia
}

\author{
Mathieu Coureuil 1,2 , Olivier Join-Lambert ${ }^{1,2,3}$, Hervé Lécuyer ${ }^{1,2,3}$, Sandrine Bourdoulous ${ }^{2,4,5}$, \\ Stefano Marullo ${ }^{2,4,5}$, and Xavier Nassif ${ }^{1,2,3}$ \\ ${ }^{1}$ INSERM, Unité U1002, Paris, France \\ ${ }^{2}$ Université Paris Descartes, Sorbonne Paris Cité, Faculté de Médecine, Paris, France \\ ${ }^{3}$ Assistance Publique-Hôpitaux de Paris, Hôpital Necker Enfants Malades, Paris, France \\ ${ }^{4}$ Inserm, U1016 Institut Cochin, Paris, France \\ ${ }^{5}$ CNRS, UMR8104 Paris, France \\ Correspondence: xavier.nassif@inserm.fr
}

\begin{abstract}
Neisseria meningitidis is responsible for two major diseases: cerebrospinal meningitis and/ or septicemia. The latter can lead to a purpura fulminans, an often-fatal condition owing to the associated septic shock. These two clinical aspects of the meningococcal infection are consequences of a tight interaction of meningococci with host endothelial cells. This interaction, mediated by the type IV pili, is responsible for the formation of microcolonies on the apical surface of the cells. This interaction is followed by the activation of signaling pathways in the host cells leading to the formation of a microbiological synapse. A low level of bacteremia is likely to favor the colonization of brain vessels, leading to bacterial meningitis, whereas the colonization of a large number of vessels by a high number of bacteria is responsible for one of the most severe forms of septic shock observed.
\end{abstract}

\begin{abstract}
Teisseria meningitidis (meningococcus) is a Wram-negative coccus restricted to humans, which is responsible for two major diseases: cerebrospinal meningitis and/or septicemia. The latter can lead to a purpura fulminans, an often-fatal condition owing to the associated septic shock. Paradoxically, N. meningitidis is a common inhabitant of the human nasopharynx, and as such is a normal, saprophytic organism that is transmitted from person to person by direct contact. Only in a small proportion of colonized subjects does the bacteria invade the bloodstream where they are responsible for septicemia and/or meningitis, after crossing of the blood-brain barrier.
\end{abstract}

Bacterial meningitis is the leading cause of central nervous system (CNS) infection. The blood-brain barrier (BBB) protects the CNS from most bacteria that may have reached the bloodstream. Most of the few types of bacteria, which can cross BBB to invade the meninges, are extracellular pathogens: Escherichia coli K1 and Streptococcus agalactiae (Group B Streptococcus) in the newborn, N. meningitidis, Haemophilus influenzae type b, and Streptococcus pneumoniae in children and adults (Pong and Bradley 1999; Huang et al. 2000; Van de Beek et al. 2006). Once inside the cerebrospinal fluid (CSF), bacterial multiplication is thought to be uncontrolled, owing to the local deficiency

Editors: Pascale Cossart and Stanley Maloy

Additional Perspectives on Bacterial Pathogenesis available at www.perspectivesinmedicine.org

Copyright (C) 2013 Cold Spring Harbor Laboratory Press; all rights reserved; doi: 10.1101/cshperspect.a012393

Cite this article as Cold Spring Harb Perspect Med 2013;3:a012393 
M. Coureuil et al.

in complement and immunoglobulins, and despite the influx of polymorphonuclear leukocytes induced by the local inflammatory response. The small number of bacterial species capable of invading the meninges suggests that specific virulence factors are required for bacteria to enter the subarachnoidal space. Among the above-mentioned extracellular bacteria, $N$. meningitidis is the pathogen that once in the bloodstream, is able to invade the meninges the most effectively. It has been estimated that $63 \%$ of the cases of bacteremia owing to N. meningitidis are associated with meningitis (InVS 2009).

The other specific clinical feature of meningococcal infection is the ability of the bacteria to multiply rapidly in the bloodstream and to be responsible for one of the most severe forms of septic shock observed, associating extensive thrombosis, vascular leakage, and cardiovascular failure (i.e., the purpura fulminans). Purpura fulminans usually occur at an early stage of the bloodstream infection, before an inflammatory response can be observed in the CSF. The epidemic nature of $N$. meningitidis infections and the high mortality rate of purpura fulminans are responsible for the fear of meningococcal infections in communities.

This article will concentrate on the pathogenesis of these two clinical aspects of the meningococcal infection.

\section{THE MENINGOCOCCAL INFECTION}

As mentioned above, N. meningitidis is a frequent asymptomatic colonizer of the human nasopharynx, and only a very small proportion of infections proceed to a sustained bacteremia. The mechanisms responsible for nasopharyngeal colonization and crossing of the nasopharyngeal mucosa remain mostly unexplained and will not be approached in this review. The reasons why disease occurs in some individuals and not in others remain unclear, but human genetic polymorphism is likely to be important in determining the outcome, particularly regarding the risk of developing purpura fulminans (Brouwer et al. 2009). In addition, all meningococci do not have the same pathogenic potential. Indeed, analysis of results from multilocus se- quence typing (MLST) has shown the existence of distinct phylogenetic groups (clonal complexes), some of which are more likely to be isolated from patients with disease than from asymptomatic carriers (Maiden et al. 1998). These are the so-called "hypervirulent" or "hyperinvasive" lineages. Recently, the presence of a prophage has been shown to be responsible for a large proportion of invasiveness of strains belonging to hyperinvasive lineages (Bille et al. $2005,2008)$. This element inserted into the bacterial chromosome can be induced to produce a filamentous phage.

N. meningitidis interacts only with human cells and there is no animal model of meningococcal sepsis. In some circumstances mice and infant rats have been used to assess the ability of the bacteria to survive in the extracellular fluids (Sun et al. 2000), but these models are unable to assess the consequences of the interaction with endothelial cells (Fig. 1).

Consequently, most hypotheses regarding the pathogenesis of meningococcal infections are derived from postmortem studies of patients who died from meningococcal meningitis or purpura fulminans or from biopsies of skin purpuric lesions (Pron et al. 1997; Harrison et al. 2002). In the brain, data obtained from a patient who died of fulminant meningoccocemia at the time bacteria were invading the CSF revealed that $N$. meningitidis adheres onto endothelial cells and forms small colonies on the endoluminal surface of the microvasculature. Bacteria are also found inside cells and in intercellular spaces (Pron et al. 1997). These data suggest that $N$. meningitidis crosses the blood-brain barrier through a direct interaction between bacteria and endothelial cells. In peripheral purpuric lesions that are commonly observed during meningococcal bacteremia, retraction of endothelial cells with capillary disruption can be observed, as well as hemorrhages, adhesion of leukocytes, and formation of small thrombi. Altogether, in vivo observations thus suggest that the pathogenesis of meningococcal infection relies on the ability of the bacteria (1) to survive and/or grow in the bloodstream, and (2) to interact and to signal to endothelial cells throughout the body. 


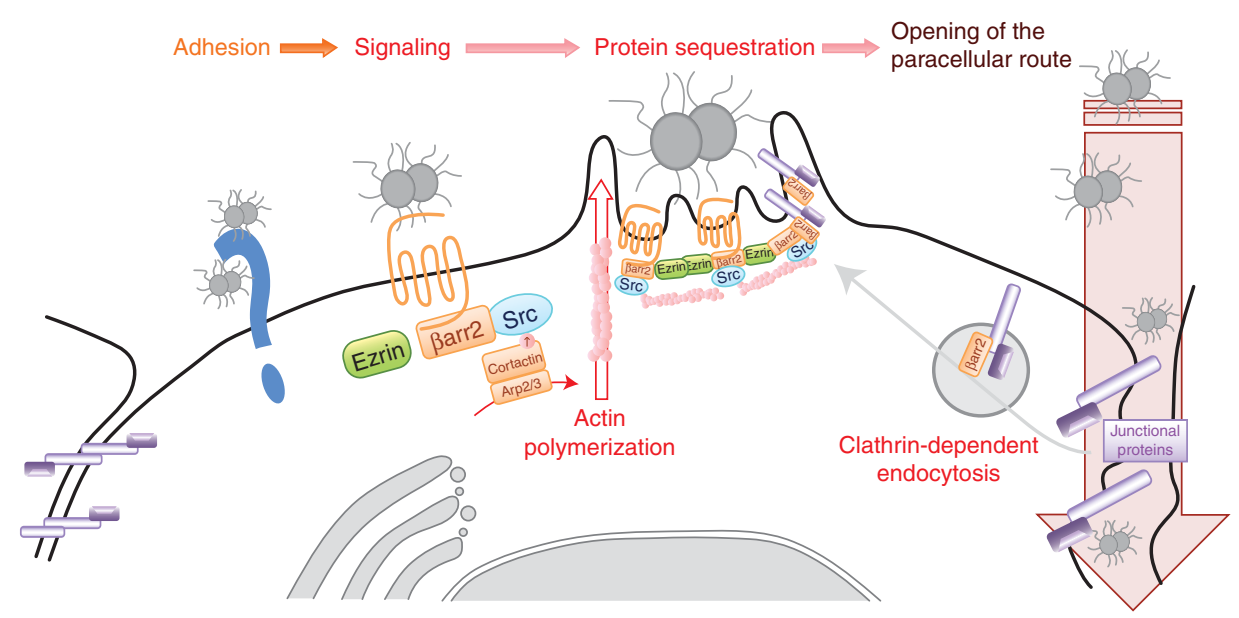

Figure 1. Interaction of Neisseria meningitidis with endothelial cells. N. meningitidis adhere to brain microvascular endothelial cells through an interaction between type IV pili and an unknown adhesion receptor (Nassif et al. 1994). Following initial bacterial adhesion, type IV pili mediate the recruitment and the activation of the $\beta 2$-adrenoceptor thus leading to the organization of a specific cytoplasmic molecular complex, referred to as cortical plaques. The formation of cortical plaques results (1) from the local accumulation of ezrin and ezrinbinding proteins, and (2) from the accumulation of $\beta$-arrestins and $\beta$-arrestin-binding molecules such as Src, which induce active actin polymerization, and that of the junctional proteins p120-catenin and VE-cadherin (Hoffmann et al. 2001; Coureuil et al. 2009, 2010). Consequently, this promotes the opening of the cell-cell junctions and allows the transmigration of bacteria through the endothelium.

From these in vivo data, it can be speculated that the clinical forms of meningococcal disease mostly reflect the level of bacteremia (Fig. 2). When a low or moderate number of meningococci circulate within in the bloodstream, the bacteria interacting with peripheral capillaries cause only few localized purpuriclesions, whereas the interaction with brain endothelial cells is sufficient to lead to meningeal invasion. In that case, meningitis is the prominent clinical sign of the disease. In contrast, in the case of high bacteremia, peripheral endothelial cells are probably massively colonized by meningococci, leading to a significant increase of vascular permeability possibly associated with extensive thrombosis and purpura. In this case, bacteria also invade the meningeal spaces, but clinical presentation of disease is a septic shock associated with purpuric lesions, purpura fulminans.

\section{THE BLOOD PHASE}

Meningeal invasion by N. meningitidis is directly correlated with bacteremia. The bactere- mia is believed to favor meningeal invasion by directly increasing the likelihood of the interaction between bacteria and the components of the blood-CSF barrier. In addition, the bacteremia is critical in the case of sepsis, as mortality is directly related to levels of bacteremia (Brandtzaeg et al. 1995). Therefore, the bacterial attributes involved in growth and/or survival in the extracellular fluids play an essential role in meningeal invasion by $N$. meningitidis and/or the sepsis outcome.

The lack of a relevant animal model has greatly hampered the identification of the bacterial factors important for bacteria survival, multiplication, or adaptation in the bloodstream. Studies aimed at identifying these factors were based on the screen of transposon mutagenesis libraries using either an infant rat model (Sun et al. 2000) or survival in complemented serum (Geoffroy et al. 2003) as readout. More recently, a transcriptomic analysis of bacteria grown in human whole blood has been performed (Echenique-Rivera et al. 2011). These studies pointed out the major role of the virulence factors 
M. Coureuil et al.

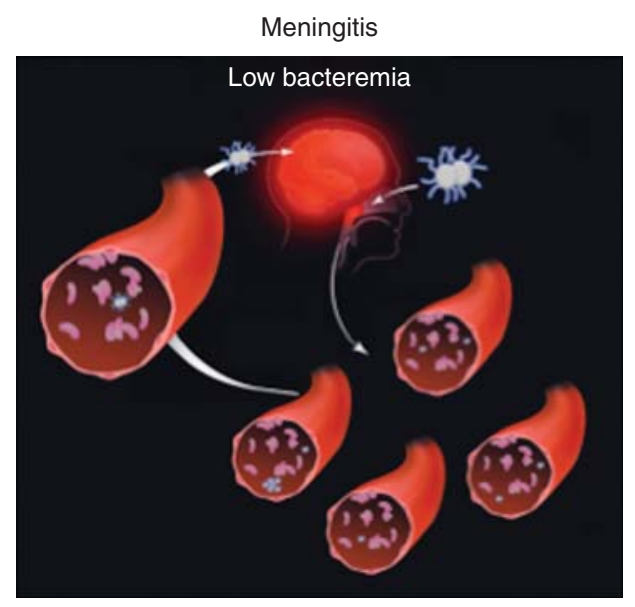

- Colonization of brain capillaries associated with uncontrolled proliferation into the CNS.

- Limited peripheral colonization associated with small purpuric lesions.

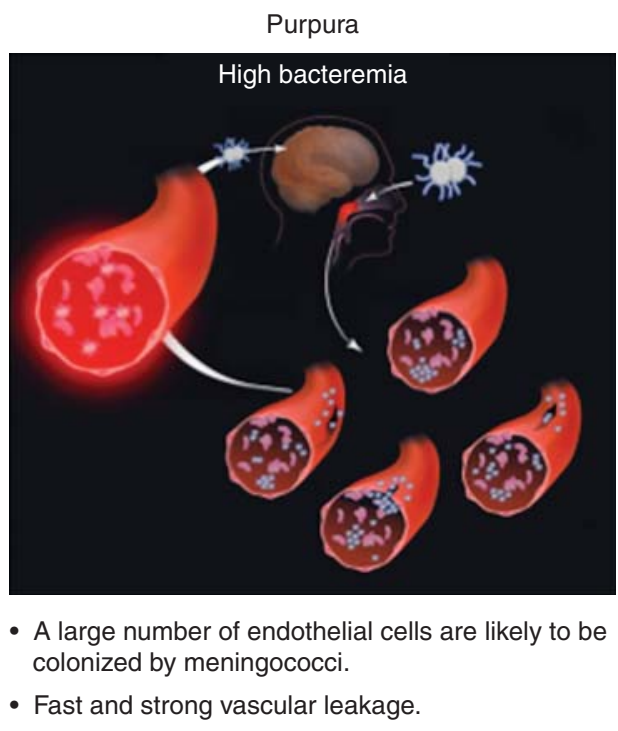

- Fast and strong vascular leakage.

Figure 2. The clinical features of meningococcemia are dependent on bacterial load. A low level of bacteremia is likely to be associated with limited vascular colonization and few purpuric lesions. Some colonies in the brain capillaries may breach the blood-brain barrier and proliferate into the central nervous system (CNS). Thus, few colonies are sufficient to induce meningitis. On the other hand, high bacteremia is associated with an important colonization of the peripheral blood vessels that results in a fast and strong vascular leakage.

commonly observed in most extracellular pathogens. In addition to iron chelation systems, which are essential for pathogenic bacteria to obtain the necessary ferric iron in vivo (Stojiljkovic et al. 1995), the polysaccharidic capsule and the lipooligosaccharide (LOS) participate in the prevention of bacterial killing by the host innate immune system effectors, such as complement. A new virulence factor, the factor$\mathrm{H}$-binding protein (Fhbp), was recently identified (Seib et al. 2008). It consists of $28 \mathrm{kDa}$ surface-exposed lipoprotein that is capable of inducing bactericidal antibodies (Masignani et al. 2003; Fletcher et al. 2004). Binding of human factor $\mathrm{H}$ to Fhbp in the bloodstream of infected individuals permits $N$. meningitidis to effectively evade host immune responses by preventing complement-mediated bacterial cell lysis. Based on sequence diversity, Fhbp proteins are classified into three variants and two subfamilies (Masignani et al. 2003; Fletcher et al. 2004). More recently, the modular protein structure has been described for Fhbp whereby a combination of five distinct variable segments de- fines six unique modular groups (Beernink and Granoff 2009). This protein is expressed by all $N$. meningitidis strains studied to date, although the level of expression varies between strains (high, intermediate, or low expressers). Antibodies directed against Fhbp are bactericidal and this protein is currently one of the best vaccine candidates (Beernink et al. 2006).

Much less is known about the contribution of other actors of the innate immune system, such as polymorphonuclear neutrophils (PMNs), in the protection against meningococcal disease. These phagocytic cells elaborate multiple mechanisms effective at killing microorganisms, such as the production of antimicrobial peptides, degradative enzymes, and reactive oxygen species derived from the oxidative burst. Recently, it has been shown that a mechanism by which $N$. meningitidis escape the killing by PMNs to survive in the bloodstream is owing to its ability to uptake available L-glutamate and to convert it to glutathione; by maintaining intracellular redox potential, this key molecule protects the bacterium from reactive 
Meningococcemia

oxygen species, such as hydrogen peroxide, that are produced by the oxidative burst of PMNs (Tala et al. 2011).

It should be pointed out that meningococcal infections are very often discovered at the stage of cerebrospinal meningitis, thus suggesting that the bloodstream phase can be totally asymptomatic until the bacteria cross the blood-brain barrier and develop an inflammation in the CSF. This is an apparent discrepancy with the fact that, in some patients, bacteria in the bloodstream lead to a purpura fulminans. As for all Gram-negative bacteria, the lipopolysaccharide is the major inducer of the proinflammatory response via activation of the Toll-like receptor 4 . This response is believed to be beneficial to the host when induced at low levels. However, it can become detrimental for the infected host when excessive stimulation occurs (Beutler and Rietschel 2003; Palsson-McDermott and O’Neill 2004; Russell 2006). Meningococcal sepsis is generally seen as the prototypical endotoxin-mediated disease. The chemical composition of the lipid A determines how well it is recognized by TLR4. N. meningitidis has been reported to produce lipid A with six acyl chains, the optimal number for TLR4 recognition. Recent data have shown that in a significant percentage of invasive meningococcal diseases, the lipid A was penta-acylated. This modification was the consequence of a mutation in the $l p X 1$ gene. The corresponding strains were dramatically less efficient in inducing the production of cytokines. Furthermore, patients infected by these strains presented significantly less frequent rash and platelet counts were higher, consistent with reduced cytokine induction and less activation of tissue-factormediated coagulopathy (Fransen et al. 2009). Because underacylation of lipid A, caused by mutations in the $l p x l 1$ gene, helps bacteria evade the innate immune system, this might explain why the blood phase could remain asymptomatic. However, such a mutation was observed in only $9 \%$ of the isolates obtained in meningococcal invasive diseases. It is therefore likely that $N$. meningitidis also has other means to reduce the activation of the host innate immune system.

\section{THE INTERACTION OF N. meningitidis WITH ENDOTHELIAL CELLS}

The tropism of N. meningitidis for microvasculature endothelial cells is a major pathophysiological issue in meningococcal infections. This property is likely to be associated with two clinical features of meningococcal infections: the crossing of the $\mathrm{BBB}$ and the formation of peripheral purpuric lesions, which can lead to a purpura fulminans. Endothelial permeability is tightly regulated to maintain endothelial integrity and to prevent exposure of the highly thrombogenic subendothelial blood vessel matrix (Danese et al. 2007). Transendothelial crossing involves both transcellular and paracellular routes. Transcellular passage requires either cell fenestration or the establishment of complex systems of vesiculo-vacuolar organelles (Nourshargh et al. 2010); it is regulated by signaling pathways involved in endocytosis and vesicular trafficking. The paracellular path is based on the coordinated opening and closure of endothelial cell-cell junctions, and is regulated via the proteins that are involved in the organization of these junctions. Endothelial cells possess specialized junctional regions such as adherens (AJs) and tight junctions (TJs). They express cell-type-specific transmembrane adhesion proteins, such as VE-cadherin at AJs, and claudin-5 at TJs. Composition and morphology of junctional structures vary along the vascular tree, as a consequence of different permeability requirements. Whereas AJs are relatively conserved in all types of vessels, TJs are lost or poorly organized where dynamic and rapid exchanges between blood and tissue are required, as in postcapillary venules. On the other hand, they are extremely well developed where permeability is strictly controlled, as required in the brain microvasculature (Dejana et al. 2009). Brain microvascular endothelial cells are essential components of the BBB. The close interaction between microvascular endothelial cells and other components of the neurovascular unit (astrocytes, pericytes, neurons, and basement membrane) ensure the differentiation of endothelial cells into brain endothelial cells and the proper function of the CNS. 
M. Coureuil et al.

Transport across the BBB is strictly limited through both physical (TJs) and metabolic barriers (enzymes, diverse transport systems) (Wolburg and Lippoldt 2002). A functional polarity exists between the luminal and basal membrane surfaces of brain microvascular endothelial cells. During bacteremia, the BBB also prevents the bacteria from invading the brain.

In vivo, blood flow generates mechanical forces that vary depending on the type and diameter of the vessels, and that could prevent bacterial interaction with the endothelial cells. The ability of N. meningitidis to bind to endothelial cells in the presence of shear stress mimicking the bloodstream was recently investigated (Mairey et al. 2006). These data revealed that, after initial attachment, bacteria have the ability to resist high blood velocities, to multiply, and to form microcolonies onto the apical surface of the endothelial cells. This resistance to shear stress and the ability to grow at the luminal surface of endothelial cells in the presence of blood flow highlight the efficacy of the interaction between $N$. meningitidis and the host cells.

Various bacterial surface components have been described allowing the interaction of $N$. meningitidis with human cells. These are type IV pili (Tfp) and other factors such as Opa or Opc proteins (for review, see Carbonnelle et al. 2009; Virji 2009). However, in capsulated bacteria, type IV pili are the main bacterial factors capable of promoting adhesion, because nonpiliated capsulated bacteria are unable to adhere to any cell type. Early work performed with piliated capsulated meningococci has shown that meningococcal interaction with human cells can be divided in two steps. The first step allows the adhesion of single diplococci in a rather inefficient manner. The second step corresponds to the bacterial division onto the apical surface of the cells. The high number of bacteria that interact with cells is a consequence of bacterial division of the few meningococci that have initially succeeded to adhere. Type IV pili are required for both steps. They promote the initial interaction of diplococci with the endothelial cells, and then generate bacteria-bacteria interactions that lead to the spreading of the bacteria on the apical surface of the cells.

\section{Type IV Pili}

\section{Pilus Biogenesis}

Type IV pili are polymeric filaments found on many Gram-negative bacteria (Wolfgang et al. 2000). These structures correspond to the multimeric assembly of a pilin subunit. Functional Tfp are dynamic structures. Pilin subunits are constantly assembled into fibers from a platform in the inner membrane. The fiber is then extruded through the outer membrane via the secretin PilQ (Wolfgang et al. 2000). A remarkable property of Tfp is their ability to retract into the bacterium from which they originate, via the action of the force-generating ATPase PilT (Merz et al. 2000; Skerker and Berg 2001). Retraction is a consequence of the disassembly of pilin subunits that are then stored in the cytoplasmic membrane (Morand et al. 2004). Tfp retraction is essential for bacterial motility (twitching motility), competence for DNA transformation, and pilus-associated signaling to host cells (Lee et al. 2005).

The major neisserial pilus subunit, the pilin, is encoded by the pile gene that is subject to antigenic variation following recombination with silent loci ( $p i l S)$. Pilin is synthesized as a preprotein. A short leader sequence (5-6 residues) is cleaved by the prepilin peptidase PilD (Lory and Strom 1997). PilD also methylates the amino-terminal phenylalanine of the mature protein product. Pilins are packed through internal hydrophobic interactions between conserved amino-terminal $\alpha$ helices, leaving hypervariable carboxy-terminal globular regions exposed (Parge et al. 1995). PilE undergoes several posttranslational modifications of serine residues including glycosylation at position 63 , whereas at position 68, the residue has been reported to be modified by phosphate, phosphoethanolamine, or phosphorylcholine addition (Forest et al. 1999; Hegge et al. 2004). Concerning the glycosylation, the structure of the sugar is different depending on the strain (Virji 1997; Chamot-Rooke et al. 2007).

In addition to PilE, pili also contain the less abundant proteins PilX, PilV, and ComP. These are called minor pilins (Winther-Larsen et al. 2001; Helaine et al. 2005; Mikaty et al. 2009; 
Brown et al. 2010) as they structurally resemble PilE and are likely to be assembled within the filaments in a similar way (Craig and Li 2008). Importantly, each minor pilin modulates Tfplinked properties (Brown et al. 2010). PilX is crucial for the formation of bacterial aggregates and indirectly controls adhesion to human cells by promoting bacteria-bacteria interactions (Helaine et al. 2005, 2007); ComP is essential for competence for DNA transformation (Wolfgang et al. 1999; Aas et al. 2002; Brown et al. 2010), whereas PilV affects several pilus-linked properties such as signaling to endothelial cells (see below) (Brown et al. 2010).

The PilC proteins play a crucial but still enigmatic role (Jonsson et al. 1991; Nassif et al. 1994; Rudel et al. 1995a,b; Wolfgang et al. 2000). Two alleles were originally discovered (Jonsson et al. 1991). Expression of both variants is subject to phase variation as a result of frameshift in homopolymeric " $G$ " tracts located in the open reading frames (Jonsson et al. 1991). PilC-null strains show impaired pilus expression and lack the ability for transformation competence. In N. meningitidis, only PilC1 is required for adhesion. PilC2, which is expressed independently of PilC1, fails to promote adhesion despite identical functions in pilus expression and transformation competence (Nassif et al. 1994; Morand et al. 2001). Abolition of pilT in a PilC-null background restores piliation, confirming the hypothesis that PilC acts as an antagonist of PilT by preventing PilT-mediated retraction (Wolfgang et al. 1998; Morand et al. 2004).

\section{Mechanism of Pilus-Mediated Interaction with Host Cells}

The molecular mechanism responsible for the first step of the adhesive process, the initial attachment of individual diplococci to the cells, is still not fully understood. One report suggested that the PilC1 protein may carry a cell-binding domain (Rudel et al. 1995b); this hypothesis was based on inhibition of adhesion using purified PilC molecules. However, nonadhesive nonpiliated isolates of a serogroup B strain with high PilC expression and piliated adhesive iso- lates with barely detectable PilC expression have been described (Virji et al. 1995). In addition, another $\mathrm{PilC}^{+} / \mathrm{PilE}^{-}$strain in which $\mathrm{PilC}$ is localized in the outer membrane, is unable to interact with eukaryotic cells (X Nassif, unpubl.), thus raising doubts on the role of PilC as an adhesin. It is therefore likely that the binding domain on the type IV pili remain to be identified. The CD46 receptor was proposed as being the adhesion receptor for both Neisseria gonorrheae and $N$. meningitidis type IV pili. However, this finding was not confirmed by subsequent studies (Kallstrom et al. 1997; Kirchner et al. 2005). The Laminin receptor was also described as a potential receptor for N. meningitidis. Two bacterial ligands for this receptor have been reported: the PilQ secretin and the PorA protein (Orihuela et al. 2009). However, considering that PilQ is expressed in nonpiliated mutants, and that nonpiliated noncapsulated strains are unable to interact with endothelial cells, its role remains to be determined. The I-domain-containing integrins were described to be essential for N. gonorrheae adhesion to primary urethral epithelial cells (Edwards and Apicella 2005). However, similar data have not been reported for $N$. meningitidis and endothelial cells.

Following the initial adhesion of a few diplococci, the formation of microcolonies on the host cells is owing to bacteria-bacteria interactions. As mentioned above, the minor pilin PilX is essential to promote interbacterial interactions, and pilX mutants, which are unable to form aggregates, are also unable to multiply at the cell surface. Bacterial spreading onto the host cells relies also on the ability of bacteria to retract their pili. Following the interactions between bacteria and target cells, in some systems, pili have been shown to retract and eventually adherent meningococci appear nonpiliated (Pujol et al. 1999; Merz et al. 2000). Measurements using optical tweezers showed that retraction of a single Tfp generates forces up to $110 \mathrm{pN}$ in a transient manner for each fiber. Bundles of Tfp, which result from the association of 8 to 10 pili, act as coordinated retractable units. Bundles can generate retraction forces in the nanonewton range (Biais 
M. Coureuil et al.

et al. 2008). The successive extension, binding, and retraction of Tfp enable bacteria to move by twitching motility and spread on the apical surface of the host cells. In addition, it has been recently shown that the addition of phosphoglycerol molecules on pilins, which is increased during bacterial cell interaction, reduces type IV pili-dependent contacts between bacteria and enhances the ability of the bacteria to detach from the adherent aggregates and to disseminate (Chamot-Rooke et al. 2011).

The Consequence of the Type IV PilusMediated Interaction with Endothelial Cells

Type IV pilus-mediated adhesion allows the activation of a signaling receptor at the site of bacterial cell interaction, which mediates the formation of a specific membrane domain designated the "cortical plaque." This membrane domain is enriched in structural proteins such as the ERM proteins (ezrin-radixin-moesin family) that anchor the actin network to the site of bacterial adhesion (Merz et al. 1999). The concomitant activation of Rho GTPases and Src tyrosine kinase promotes cortactindependent actin polymerization (Coureuil et al. 2009). In addition to the accumulation of the structural proteins, the cortical plaque is enriched in adhesion receptors or membrane receptors of receptor tyrosine kinase and G-protein-coupled receptor (GPCR) families (Hoffmann et al. 2001; Doulet et al. 2006; personal observation).

The interaction domain between the bacterial colony and the host cell can be compared to a synapse because (1) it is composed of adhesion receptors and signaling receptors (that are mostly immunoglobulin domain-containing receptors and GPCRs, respectively), and (2) it transmits signals. One particularity of this "bacterial synapse" is that adhesion receptor and signaling receptor are not internalized but sequestrated underneath the meningococcal colonies (Doulet et al. 2006; personal observation). The "bacterial synapse" is maintained by a cortical cytoskeletal protein network highly enriched in members of the ERM protein family, which are anchored to the plasma membrane by their PIP2-binding domain and control the organization of the cortical actin cytoskeleton through their carboxy-terminal F-actin binding sites (Eugene et al. 2002; Lambotin et al. 2005; Doulet et al. 2006). ERM proteins also bind the cytoplasmic domain of several ERM-binding transmembrane receptors such as CD44 and ICAM-1 through their carboxy-terminal domain (Merz and So 1997; Merz et al. 1999; Eugene et al. 2002). As a consequence of the recruitment of ERM proteins, many receptors known to be involved in leukocyte adhesion are sequestered underneath bacterial colonies (Doulet et al. 2006). During leukocyte adhesion, these components that form the "endothelial docking structures" or "transmigratory cups" are essential to promote firm adhesion and extravasation of leukocytes through the endothelium. Transmigratory cups result from the dynamic redistribution of ICAM-1, VCAM-1, E-selectin, and CD44 at the endothelial-leukocyte contact area, accompanied by the recruitment of activated ERM proteins, and leads to cortical actin polymerization (Carman and Springer 2004; Shaw et al. 2004; Barreiro et al. 2005). Because the same set of endothelial proteins is present within the membrane protrusions induced by N. meningitidis and within the docking structures promoted by leukocyte adhesion, it was suggested that $N$. meningitidis highjack the leukocyte adhesion pathway (Doulet et al. 2006).

\section{The $\beta 2$ Adrenergic Receptor Is the Signaling Receptor}

The initial cellular receptor responsible for pilus-mediated adhesion remains unknown. However, the $\beta 2$-adrenergic receptor was recently shown to be recruited at the site of bacterial cell interaction following this initial event and was described as an important signaling receptor for N. meningitidis (Coureuil et al. 2010; Lecuyer et al. 2012). The $\beta 2$-adrenergic receptor is a GPCR that signals via the heterotrimeric Gos protein and $\beta$-arrestins. This receptor is also known for its implication in vascular homeostasis and disease. The expression of both the $\beta 2$ adrenergic receptor and $\beta$-arrestins is sufficient 
to promote an N. meningitides-induced cell response in an incompetent cell line, such as HEK293 cells (Coureuil et al. 2010). It has been shown that PilE and PilV, the major pilin subunit and minor pilin subunit, respectively, directly interact with the extracellular aminoterminal domain of the $\beta 2$-adrenergic receptor to transmit the signal (Coureuil et al. 2010). This interaction is believed to modify the conformation of the receptor, resulting in the activation of $\beta$-arrestin-mediated signaling without activating the heterotrimeric $\mathrm{G} \alpha$ s protein and the downstream adenyl cyclase/cAMP pathway, a property referred to as biased activation (Coureuil et al. 2010). $\beta$-arrestins are scaffolding proteins involved in many cellular processes such as receptor internalization and actin polymerization (DeWire et al. 2007). Following the activation of the $\beta 2$-adrenergic receptor by $N$. meningitidis and the accumulation of $\beta$-arrestins, the signal leads to the formation of a "raftlike" membrane domain enriched in cholesterol and PIP2, in which several transmembrane receptors and structural proteins are sequestered, thus leading to the subsequent formation of the cortical plaque (Doulet et al. 2006; Mikaty et al. 2009). It is likely that accumulated $\beta$-arrestins underneath bacteria play a major role in the sequestration of these signaling molecules. Nevertheless, inhibition of the $\beta 2$-adrenergic-mediated signaling does not affect bacterial adhesion (Coureuil et al. 2010), thus confirming that adhesion and signaling are two independent events, owing to the recognition of two separate receptors.

In summary, following the initial adhesion of the bacteria to a yet-unknown receptor, the $\beta 2$-adrenergic receptor is recruited and activated by components of the type IV pili, thus inducing the formation of the cortical plaque.

\section{Consequences of $N$. meningitidis-Induced Signaling on Endothelial Cells}

The formation of the cortical plaque is accompanied by a robust local stimulation of actin polymerization leading to the elongation of membrane protrusions around bacteria (Merz and So 1997; Eugene et al. 2002). Interestingly, the formation of such protrusions was also observed in vivo on brain endothelial cells by transmission electron microscopy analysis of brain sections from a child who died of fulminant meningoccemia (Pujol et al. 1997). These cellular projections have been shown to be required to allow the microcolonies to stand up to the shear stress of the bloodstream (Mairey et al. 2006). Actin polymerization relies on the activation of the small GTPases of the Rho family (Eugene et al. 2002; Lambotin et al. 2005). In addition, it has been shown that proper actin polymerization in these membrane protrusions relies on cortactin phosphorylation. Cortactin (or cortical actin-binding protein) is a perinuclear cytoplasmic protein that is involved in the reorganization of the cell cortical actin cytoskeleton. It appears that cortactin recruitment and phosphorylation is finely tuned by N. meningitidis:

1. The recruitment of cortactin at the site of $N$. meningitidis adhesion is controlled by the Cdc42-Par6/PKCל pathway (Coureuil et al. 2009).

2. The activation of cortactin through phosphorylation is controlled by the tyrosine kinase Src that is sequestered in the cortical plaque and activated by direct interaction with $\beta$-arrestins (Luttrell et al. 1999; Coureuil et al. 2010; Lecuyer et al. 2012).

3. The ErbB2 tyrosine kinase receptor also regulates Src activity and the subsequent cortactin phosphorylation (Hoffmann et al. 2001). The ErbB2 tyrosine kinase receptor belongs to the family of epidermal growth factor (EGF) receptors. The interaction of N. meningitidis with human endothelial cells leads to ErbB2 activation in the cortical plaque most likely via homodimerization. This is an example of secondary signaling activated by the accumulation of a cellular receptor at the site of bacterial adhesion that contributes to the formation of the cortical plaque.

Another consequence of the formation of the cortical plaque is the opening of the interendothelial junctions allowing the transmigration of bacteria through a paracellular route. Indeed, it has been shown that $N$. meningitidis 
M. Coureuil et al.

recruits junctional proteins underneath the colony into the cortical plaque. This recruitment is owing to the activation of the Cdc42-Par3/ Par6/PKC $\zeta$ pathway that is usually involved in the formation of adherens and TJs at cell-cell contact. Here, the ectopic activation of the polarity complex Par3/Par6/PKCל leads to abnormal recruitment of junctional proteins that are sequestrated underneath bacterial colonies through their interaction with $\beta$-arrestins. As a consequence, these molecules are depleted at the intercellular junctions causing endothelium leakage. Adhesion of N. meningitidis lately promotes the cleavage of occludin (a component of the tight junction) by the metalloproteinase MMP-8 (Schubert-Unkmeir et al. 2010), further altering the intercellular junctions.

In summary, two important consequences for meningococcal pathogenesis are associated with type IV pilus-mediated adhesion: (1) the ability to be protected from the shear stress by the formation of a microvilli-like structure on the apical surface of endothelial cells, which is a prerequisite to the formation of adhesive bacterial microcolonies, and (2) the retraction of the opening of a paracellular route between endothelial cells, thus locally jeopardizing the integrity of the capillaries.

\section{CONSEQUENCES OF N. meningitidis INTERACTION WITH ENDOTHELIAL CELLS FOR MENINGOCOCCAL PATHOGENESIS}

As described above, N. meningitidis is an extracellular pathogen that once disseminated into the bloodstream, has two specificities: (1) its ability to interact freely with the BBB to reach the subarachnoidal space, and (2) in some circumstances to be responsible for a severe shock with extensive purpura (i.e., the purpura fulminans).

\section{The Crossing of the BBB}

Following its interaction of bacteria with endothelial cells, there are at least four strategies for a pathogen to cross a monolayer of brain endothelial cell monolayers: (1) transcellular transport by passive or adhesion-induced transcyto- sis, (2) paracellular passage through open TJs, (3) disruption of the endothelial barrier owing to a direct cytotoxic effect, and (4) leukocytefacilitated transport by infected phagocytes.

Extracellular pathogens, such as N. meningitidis, do not use leukocytes as vehicles to cross the blood-CSF barrier. A breakdown of the blood-CNS barrier owing to apoptosis or bacterial cytotoxity is unlikely, because tissue lesions, such as hemorrhages in the subarachnoidal space, are uncommon during bacterial meningitis. Therefore, the entry of blood-borne pathogen meningococcus most probably respects the architecture of the blood-CNS barrier (Koedel et al. 2002). Accordingly, adhesion of $N$. meningitidis to endothelial cells can induce an intracellular signaling pathway leading to disruption of intercellular TJs or, alternatively, bacteria may induce their own transcytosis through the cell monolayer. In vitro, transcytosis of bacteria through human brain endothelial cells has been shown for S. pneumoniae (Ring et al. 1998), E. coli (Prasadarao et al. 1999; Stins et al. 1999, 2001), S. agalactiae (Nizet et al. 1997), and through human umbilical vein endothelial cells for $H$. influenzae (Virji et al. 1991, 1992). Although N. meningitidis can readily be internalized in vitro within vacuoles in human brain microvascular endothelial cells (Nikulin et al. 2006), the fact that $N$. meningitidis can open gaps in a monolayer of brain endothelial cells as a consequence of the delocalization of junctional components suggests that $N$. meningitidis cross the $\mathrm{BBB}$ using the paracellular route.

\section{The Purpura Fulminans}

In purpuric lesions, endothelial cell retraction is observed at the site of bacterial endothelial cells interaction, associated with a loss of the integrity of the capillary resulting in an increase in permeability, hemorrhages, leukocyte aggregation and formation of thrombi hemorrhages, and adhesion of leukocytes (Dupin et al. 2012). As the signaling events induced by adherent microcolonies of $N$. meningitidis are responsible for disjunction of endothelial cells in vitro, it is most likely that these lesions are also owing to initiation of similar signaling pathways in 
vivo. The colonization of a large number of vessels by a high number of bacteria as those seen in purpura fulminans and the consequences of the corresponding signaling may explain the extensive purpuric lesions and thrombosis as well as the loss of vascular integrity responsible for the severity of the shock.

Recent exciting findings have considerably expanded our understanding of the cellular events involved in meningococcal interaction with endothelial cells, thus leading to the establishment of a hypothesis regarding the pathogenesis of meningeal invasion and of the purpura. However, despite recent advances in our understanding of these molecular mechanisms, much remains to be discovered about the complex molecular networks involved. Among the major issues is the identification of the receptor for meningococcal adhesion, which would constitute a significant breakthrough in the field, and the identification of animal models that would allow one to confirm the in vitro observations.

\section{ACKNOWLEDGMENTS}

The laboratory of X.N. is supported by INSERM, Université Paris Descartes, a grant from "La Fondation pour la Recherche Médicale," and ANR Grant No. ANR-AAP-2009-0626. M.C. is supported by an ANR Grant No. ANR-11-JSV3-0002 and a grant "Mairie de Paris-Programme EMERGENCE.”

\section{REFERENCES}

Aas FE, Wolfgang M, Frye S, Dunham S, Lovold C, Koomey M. 2002. Competence for natural transformation in Neisseria gonorrhoeae: Components of DNA binding and uptake linked to type IV pilus expression. Mol Microbiol 46: 749-760.

Barreiro O, Yanez-Mo M, Sala-Valdes M, Gutierrez-Lopez MD, Ovalle S, Higginbottom A, Monk PN, Cabanas C, Sanchez-Madrid F. 2005. Endothelial tetraspanin microdomains regulate leukocyte firm adhesion during extravasation. Blood 105: 2852-2861.

Beernink PT, Granoff DM. 2009. The modular architecture of meningococcal factor H-binding protein. Microbiology 155: 2873-2883.

Beernink PT, Leipus A, Granoff DM. 2006. Rapid genetic grouping of factor h-binding protein (genome-derived neisserial antigen 1870), a promising group B menin- gococcal vaccine candidate. Clin Vaccine Immunol 13: $758-763$.

Beutler B, Rietschel ET. 2003. Innate immune sensing and its roots: The story of endotoxin. Nat Rev Immunol 3: $169-176$.

Biais N, Ladoux B, Higashi D, So M, Sheetz M. 2008. Cooperative retraction of bundled type IV pili enables nanonewton force generation. PLoS Biol 6: e87.

Bille E, Zahar JR, Perrin A, Morelle S, Kriz P, Jolley KA, Maiden MC, Dervin C, Nassif X, Tinsley CR. 2005. A chromosomally integrated bacteriophage in invasive meningococci. J Exp Med 201: 1905-1913.

Bille E, Ure R, Gray SJ, Kaczmarski EB, McCarthy ND, Nassif X, Maiden MC, Tinsley CR. 2008. Association of a bacteriophage with meningococcal disease in young adults. PloS ONE 3: e3885.

Brandtzaeg P, Ovstebo R, Kierulf P. 1995. Bacteremia and compartmentalization of LPS in meningococcal disease. Prog Clin Biol Res 392: 219-233.

Brouwer MC, de Gans J, Heckenberg SGB, Zwinderman AH, van der Poll T, van de Beek D. 2009. Host genetic susceptibility to pneumococcal and meningococcal disease: A systematic review and meta-analysis. Lancet Infect Dis 9: $31-44$.

Brown DR, Helaine S, Carbonnelle E, Pelicic V. 2010. Systematic functional analysis reveals that a set of seven genes is involved in fine-tuning of the multiple functions mediated by type IV Pili in Neisseria meningitidis. Infect Immun 78: 3053-3063.

Carbonnelle E, Hill DJ, Morand P, Griffiths NJ, Bourdoulous S, Murillo I, Nassif X, Virji M. 2009. Meningococcal interactions with the host. Vaccine 27 (Suppl 2): B78-B89.

Carman CV, Springer TA. 2004. A transmigratory cup in leukocyte diapedesis both through individual vascular endothelial cells and between them. J Cell Biol 167: 377-388.

Chamot-Rooke J, Rousseau B, Lanternier F, Mikaty G, Mairey E, Malosse C, Bouchoux G, Pelicic V, Camoin L, Nassif X, et al. 2007. Alternative Neisseria spp. type IV pilin glycosylation with a glyceramido acetamido trideoxyhexose residue. Proc Natl Acad Sci 104: 1478314788 .

Chamot-Rooke J, Mikaty G, Malosse C, Soyer M, Dumont A, Gault J, Imhaus AF, Martin P, Trellet M, Clary G, et al. 2011. Posttranslational modification of pili upon cell contact triggers $N$. meningitidis dissemination. Science 331: 778-782.

Coureuil M, Mikaty G, Miller F, Lecuyer H, Bernard C, Bourdoulous S, Dumenil G, Mege RM, Weksler BB, Romero IA, et al. 2009. Meningococcal type IV Pili recruit the polarity complex to cross the brain endothelium. Science 25: 83-87.

Coureuil M, Lecuyer H, Scott MG, Boularan C, Enslen H, Soyer M, Mikaty G, Bourdoulous S, Nassif X, Marullo S. 2010. Meningococcus hijacks a $\beta 2$-adrenoceptor $/ \beta$-arrestin pathway to cross brain microvasculature endothelium. Cell 143: 1149-1160.

Craig L, Li J. 2008. Type IV pili: Paradoxes in form and function. Curr Opin Struc Biol 18: 267-277. 
M. Coureuil et al.

Danese S, Dejana E, Fiocchi C. 2007. Immune regulation by microvascular endothelial cells: Directing innate and adaptive immunity, coagulation, and inflammation. $J$ Immunol 178: 6017-6022.

Dejana E, Tournier-Lasserve E, Weinstein BM. 2009. The control of vascular integrity by endothelial cell junctions: Molecular basis and pathological implications. Dev Cell 16: $209-221$.

DeWire SM, Ahn S, Lefkowitz RJ, Shenoy SK. 2007. $\beta$-arrestins and cell signaling. Ann Rev Physiol 69: 483-510.

Doulet N, Donnadieu E, Laran-Chich MP, Niedergang F Nassif X, Couraud PO, Bourdoulous S. 2006. Neisseria meningitidis infection of human endothelial cells interferes with leukocyte transmigration by preventing the formation of endothelial docking structures. J Cell Biol 173: $627-637$.

Dupin N, Lecuyer H, Carlotti A, Poyart C, Coureuil M, Chanal J, Schmitt A, Vacher-Lavenu MC, Taha MK, Nassif X, et al. 2012. Chronic meningoccemia involves perivascular invasion by N. meningitidis through the remodelling of endothelial barriers. Clin Infect Dis 54: $1162-1165$.

Echenique-Rivera H, Muzzi A, Del Tordello E, Seib KL, Francois P, Rappuoli R, Pizza M, Serruto D. 2011. Transcriptome analysis of Neisseria meningitidis in human whole blood and mutagenesis studies identify virulence factors involved in blood survival. PLoS Pathog 7: e1002027.

Edwards JL, Apicella MA. 2005. I-domain-containing integrins serve as pilus receptors for Neisseria gonorrhoeae adherence to human epithelial cells. Cell Microbiol 7: 1197-1211.

Eugene E, Hoffmann I, Pujol C, Couraud PO, Bourdoulous S, Nassif X. 2002. Microvilli-like structures are associated with the internalization of virulent capsulated Neisseria meningitidis into vascular endothelial cells. J Cell Sci 115: 1231-1241.

Fletcher LD, Bernfield L, Barniak V, Farley JE, Howell A, Knauf M, Ooi P, Smith RP, Weise P, Wetherell M, et al 2004. Vaccine potential of the Neisseria meningitidis 2086 lipoprotein. Infect Immun 72: 2088-2100.

Forest KT, Dunham SA, Koomey M, Tainer JA. 1999. Crystallographic structure reveals phosphorylated pilin from Neisseria: Phosphoserine sites modify type IV pilus surface chemistry and fibre morphology. Mol Microbiol 31: 743-752.

Fransen F, Heckenberg SG, Hamstra HJ, Feller M, Boog CJ, van Putten JP, van de Beek D, van der Ende A, van der Ley P. 2009. Naturally occurring lipid A mutants in Neisseria meningitidis from patients with invasive meningococcal disease are associated with reduced coagulopathy PLoS Pathog 5: e1000396.

Geoffroy MC, Floquet S, Metais A, Nassif X, Pelicic V. 2003. Large-scale analysis of the meningococcus genome by gene disruption: Resistance to complement-mediated lysis. Genome Res 13: 391-398.

Harrison OB, Robertson BD, Faust SN, Jepson MA, Goldin RD, Levin M, Heyderman RS. 2002. Analysis of pathogen-host cell interactions in purpura fulminans: Expression of capsule, type IV pili, and PorA by Neisseria meningitidis in vivo. Infect Immun 70: 5193-5201.
Hegge FT, Hitchen PG, Aas FE, Kristiansen H, Lovold C, Egge-Jacobsen W, Panico M, Leong WY, Bull V, Virji M, et al. 2004. Unique modifications with phosphocholine and phosphoethanolamine define alternate antigenic forms of Neisseria gonorrhoeae type IV pili. Proc Natl Acad Sci 101: 10798-10803.

Helaine S, Carbonnelle E, Prouvensier L, Beretti JL, NassifX, Pelicic V. 2005. PilX, a pilus-associated protein essential for bacterial aggregation, is a key to pilus-facilitated attachment of Neisseria meningitidis to human cells. Mol Microbiol 55: 65-77.

Helaine S, Dyer DH, Nassif X, Pelicic V, Forest KT. 2007. 3D structure/function analysis of PilX reveals how minor pilins can modulate the virulence properties of type IV pili. Proc Natl Acad Sci 104: 15888-15893.

Hoffmann I, Eugene E, Nassif X, Couraud PO, Bourdoulous S. 2001. Activation of ErbB2 receptor tyrosine kinase supports invasion of endothelial cells by Neisseria meningitidis. J Cell Biol 155: 133-143.

Huang SH, Stins MF, Kim KS. 2000. Bacterial penetration across the blood-brain barrier during the development of neonatal meningitis. Microbes Infect 2: 1237-1244.

In VS. 2009. Réseau EPIBAC. Surveillance des infections invasives à Haemophilus influenzae, Listeria monocytogenes, Neisseria meningitidis, Streptococcus pneumoniae, Streptococcus agalactiae (B) et Streptococcus pyogenes (A) en France métropolitaine. Institut National de Veille Sanitaire (InVs), Paris.

Jonsson AB, Nyberg G, Normark S. 1991. Phase variation of gonococcal pili by frameshift mutation in pilC, a novel gene for pilus assembly. EMBO J 10: 477-488.

Kallstrom H, Liszewski MK, Atkinson JP, Jonsson AB. 1997. Membrane cofactor protein (MCP or CD46) is a cellular pilus receptor for pathogenic Neisseria. Mol Microbiol 25: 639-647.

Kirchner M, Heuer D, Meyer TF. 2005. CD46-independent binding of neisserial type IV pili and the major pilus adhesin, PilC, to human epithelial cells. Infect Immun 73: $3072-3082$.

Koedel U, Scheld WM, Pfister HW. 2002. Pathogenesis and pathophysiology of pneumococcal meningitis. Lancet Infect Dis 2: 721-736.

Lambotin M, Hoffmann I, Laran-Chich MP, Nassif X, Couraud PO, Bourdoulous S. 2005. Invasion of endothelial cells by Neisseria meningitidis requires cortactin recruitment by a phosphoinositide-3-kinase/Racl signalling pathway triggered by the lipo-oligosaccharide. J Cell Sci 118: 3805-3816.

Lecuyer H, Nassif X, Coureuil M. 2012. Two strikingly different signaling pathways are induced by Meningococcal type IV pili on endothelial and epithelial cells. Infect Immun 80: $175-186$

Lee SW, Higashi DL, Snyder A, Merz AJ, Potter L, So M. 2005. PilT is required for PI(3,4,5)P3-mediated crosstalk between Neisseria gonorrhoeae and epithelial cells. Cell Microbiol 7: 1271-1284.

Lory S, Strom MS. 1997. Structure-function relationship of type-IV prepilin peptidase of Pseudomonas aeruginosaA review. Gene 192: 117-121.

Luttrell LM, Ferguson SS, Daaka Y, Miller WE, Maudsley S, Della Rocca GJ, Lin F, Kawakatsu H, Owada K, Luttrell DK, et al. 1999. $\beta$-arrestin-dependent formation 
of $\beta 2$ adrenergic receptor-Src protein kinase complexes. Science 283: 655-661.

Maiden MC, Bygraves JA, Feil E, Morelli G, Russell JE, Urwin R, Zhang Q, Zhou J, Zurth K, Caugant DA, et al. 1998. Multilocus sequence typing: A portable approach to the identification of clones within populations of pathogenic microorganisms. Proc Natl Acad Sci 95: 3140-3145.

Mairey E, Genovesio A, Donnadieu E, Bernard C, Jaubert F Pinard E, Seylaz J, Olivo-Marin JC, Nassif X, Dumenil G. 2006. Cerebral microcirculation shear stress levels determine Neisseria meningitidis attachment sites along the blood-brain barrier. J Exp Med 203: 1939-1950.

Masignani V, Comanducci M, Giuliani MM, Bambini S, Adu-Bobie J, Arico B, Brunelli B, Pieri A, Santini L, Savino S, et al. 2003. Vaccination against Neisseria meningitidis using three variants of the lipoprotein GNA1870. J Exp Med 197: 789-799.

Merz AJ, So M. 1997. Attachment of piliated, Opa- and Opcgonococci and meningococci to epithelial cells elicits cortical actin rearrangements and clustering of tyrosinephosphorylated proteins. Infect Immun 65: 4341-4349.

Merz AJ, Enns CA, So M. 1999. Type IV pili of pathogenic Neisseriae elicit cortical plaque formation in epithelial cells. Mol Microbiol 32: 1316-1332.

Merz AJ, So M, Sheetz MP. 2000. Pilus retraction powers bacterial twitching motility. Nature 407: 98-102.

Mikaty G, Soyer M, Mairey E, Henry N, Dyer D, Forest KT, Morand P, Guadagnini S, Prevost MC, Nassif X, et al 2009. Extracellular bacterial pathogen induces host cell surface reorganization to resist shear stress. PLoS Pathog 5: e1000314.

Morand PC, Tattevin P, Eugene E, Beretti JL, Nassif X. 2001. The adhesive property of the type IV pilus-associated component PilC1 of pathogenic Neisseria is supported by the conformational structure of the $\mathrm{N}$-terminal part of the molecule. Mol Microbiol 40: 846-856.

Morand PC, Bille E, Morelle S, Eugene E, Beretti JL, Wolfgang M, Meyer TF, Koomey M, Nassif X. 2004. Type IV pilus retraction in pathogenic Neisseria is regulated by the PilC proteins. EMBO J 23: 2009-2017.

Nassif X, Beretti JL, Lowy J, Stenberg P, O'Gaora P, Pfeifer J, Normark S, So M. 1994. Roles of pilin and PilC in adhesion of Neisseria meningitidis to human epithelial and endothelial cells. Proc Natl Acad Sci 91: 3769-3773.

Nikulin J, Panzner U, Frosch M, Schubert-Unkmeir A. 2006. Intracellular survival and replication of Neisseria meningitidis in human brain microvascular endothelial cells. Int J Med Microbiol 296: 553-558.

Nizet V, Kim KS, Stins M, Jonas M, Chi EY, Nguyen D, Rubens CE. 1997. Invasion of brain microvascular endothelial cells by group B streptococci. Infect Immun 65: 5074-5081.

Nourshargh S, Hordijk PL, Sixt M. 2010. Breaching multiple barriers: Leukocyte motility through venular walls and the interstitium. Nat Rev 11: 366-378.

Orihuela CJ, Mahdavi J, Thornton J, Mann B, Wooldridge KG, Abouseada N, Oldfield NJ, Self T, Ala'Aldeen DA, Tuomanen EI. 2009. Laminin receptor initiates bacterial contact with the blood brain barrier in experimental meningitis models. J Clin Invest 119: 1638-1646.
Palsson-McDermott EM, O’Neill LA. 2004. Signal transduction by the lipopolysaccharide receptor, Toll-like receptor-4. Immunology 113: 153-162.

Parge HE, Forest KT, Hickey MJ, Christensen DA, Getzoff ED, Tainer JA. 1995. Structure of the fibre-forming protein pilin at 2.6 A resolution. Nature 378: 32-38.

Pong A, Bradley JS. 1999. Bacterial meningitis and the newborn infant. Infect Dis Clin North Am 13: 711-733, viii.

Prasadarao NV, Wass CA, Stins MF, Kim KS. 1999. Outer membrane protein A-promoted actin condensation of brain microvascular endothelial cells is required for $E s-$ cherichia coli invasion. Infect Immun 67: 5775-5783.

Pron B, Taha MK, Rambaud C, Fournet JC, Pattey N, Monnet JP, Musilek M, Beretti JL, Nassif X. 1997. Interaction of Neisseria meningitidis with the components of the blood-brain barrier correlates with an increased expression of PilC. J Infect Dis 176: 1285-1292.

Pujol C, Eugene E, de Saint Martin L, Nassif X. 1997. Interaction of Neisseria meningitidis with a polarized monolayer of epithelial cells. Infect Immun 65: 4836-4842.

Pujol C, Eugene E, Marceau M, Nassif X. 1999. The meningococcal PilT protein is required for induction of intimate attachment to epithelial cells following pilus-mediated adhesion. Proc Natl Acad Sci 96: 4017-4022.

Ring A, Weiser JN, Tuomanen EI. 1998. Pneumococcal trafficking across the blood-brain barrier. Molecular analysis of a novel bidirectional pathway. J Clin Invest 102: 347-360.

Rudel T, Facius D, Barten R, Scheuerpflug I, Nonnenmacher E, Meyer TF. 1995a. Role of pili and the phasevariable PilC protein in natural competence for transformation of Neisseria gonorrhoeae. Proc Natl Acad Sci 92: 7986-7990.

Rudel T, Scheurerpflug I, Meyer TF. 1995b. Neisseria PilC protein identified as type- 4 pilus tip-located adhesin. Nature 373: 357-359.

Russell JA. 2006. Management of sepsis. N Engl J Med 355: 1699-1713.

Schubert-Unkmeir A, Konrad C, Slanina H, Czapek F, Hebling S, Frosch M. 2010. Neisseria meningitidis induces brain microvascular endothelial cell detachment from the matrix and cleavage of occludin: A role for MMP-8. PLoS Pathog 6: e1000874.

Seib KL, Serruto D, Oriente F, Delany I, Adu-Bobie J, Veggi D, Arico B, Rappuoli R, Pizza M. 2008. Factor Hbinding protein is important for meningococcal survival in human whole blood and serum, and in the presence of the antimicrobial peptide LL-37. Infect Immun 77: 292-299.

Shaw SK, Ma S, Kim MB, Rao RM, Hartman CU, Froio RM, Yang L, Jones T, Liu Y, Nusrat A, et al. 2004. Coordinated redistribution of leukocyte LFA-1 and endothelial cell ICAM-1 accompany neutrophil transmigration. $J$ Exp Med 200: 1571-1580.

Skerker JM, Berg HC. 2001. Direct observation of extension and retraction of type IV pili. Proc Natl Acad Sci 98: 6901-6904.

Stins MF, Nemani PV, Wass C, Kim KS. 1999. Escherichia coli binding to and invasion of brain microvascular 
M. Coureuil et al.

endothelial cells derived from humans and rats of different ages. Infect Immun 67: 5522-5525.

Stins MF, Badger J, Sik Kim K. 2001. Bacterial invasion and transcytosis in transfected human brain microvascular endothelial cells. Microb Pathog 30: 19-28.

Stojiljkovic I, Hwa V, de Saint Martin L, O'Gaora P, Nassif X, Heffron F, So M. 1995. The Neisseria meningitidis haemoglobin receptor: Its role in iron utilization and virulence. Mol Microbiol 15: 531-541.

Sun YH, Bakshi S, Chalmers R, Tang CM. 2000. Functional genomics of Neisseria meningitidis pathogenesis. Nat Med 6: 1269-1273.

Tala A, Monaco C, Nagorska K, Exley RM, Corbett A, Zychlinsky A, Alifano P, Tang CM. 2011. Glutamate utilization promotes meningococcal survival in vivo through avoidance of the neutrophil oxidative burst. Mol Microbiol 81: 1330-1342.

Van de Beek D, de Gans J, Tunkel AR, Wijdicks EF. 2006. Community-acquired bacterial meningitis in adults. N Engl J Med 354: 44-53.

Virji M. 1997. Post-translational modifications of meningococcal pili. Identification of common substituents: Glycans and $\alpha$-glycerophosphate-A review. Gene 192: 141147.

Virji M. 2009. Pathogenic neisseriae: Surface modulation, pathogenesis and infection control. Nat Rev Microbiol 7: 274-286.

Virji M, Kayhty H, Ferguson DJ, Alexandrescu C, Moxon ER. 1991. Interactions of Haemophilus influenzae with cultured human endothelial cells. Microb Pathog 10: 231245.
Virji M, Kayhty H, Ferguson DJ, Alexandrescu C, Moxon ER 1992. Interactions of Haemophilus influenzae with human endothelial cells in vitro. J Infect Dis 165 (Suppl 1): S115S116.

Virji M, Makepeace K, Peak I, Payne G, Saunders JR, Ferguson DJ, Moxon ER. 1995. Functional implications of the expression of PilC proteins in meningococci. Mol Microbiol 16: 1087-1097.

Winther-Larsen HC, Hegge FT, Wolfgang M, Hayes SF, van Putten JP, Koomey M. 2001. Neisseria gonorrhoeae PilV, a type IV pilus-associated protein essential to human epithelial cell adherence. Proc Natl Acad Sci 98: 1527615281.

Wolburg H, Lippoldt A. 2002. Tight junctions of the bloodbrain barrier: Development, composition and regulation. Vascul Pharmacol 38: 323-337.

Wolfgang M, Lauer P, Park HS, Brossay L, Hebert J, Koomey M. 1998. PilT mutations lead to simultaneous defects in competence for natural transformation and twitching motility in piliated Neisseria gonorrhoeae. Mol Microbiol 29: 321-330.

Wolfgang M, van Putten JP, Hayes SF, Koomey M. 1999. The comP locus of Neisseria gonorrhoeae encodes a type IV prepilin that is dispensable for pilus biogenesis but essential for natural transformation. Mol Microbiol 31: 1345-1357.

Wolfgang M, van Putten JP, Hayes SF, Dorward D, Koomey M. 2000. Components and dynamics of fiber formation define a ubiquitous biogenesis pathway for bacterial pili. $E M B O J$ J 19: 6408-6418. 


\section{$\&_{\mathrm{CSH}}^{\infty} \&$ Cold Spring Harbor

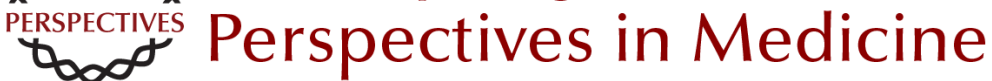

\section{Pathogenesis of Meningococcemia}

Mathieu Coureuil, Olivier Join-Lambert, Hervé Lécuyer, Sandrine Bourdoulous, Stefano Marullo and Xavier Nassif

Cold Spring Harb Perspect Med 2013; doi: 10.1101/cshperspect.a012393

\section{Subject Collection Bacterial Pathogenesis}

Therapeutic and Prophylactic Applications of Bacteriophage Components in Modern Medicine Sankar Adhya, Carl R. Merril and Biswajit Biswas

Vaccines, Reverse Vaccinology, and Bacterial Pathogenesis Isabel Delany, Rino Rappuoli and Kate L. Seib

Helicobacter and Salmonella Persistent Infection Strategies Denise M. Monack

Echoes of a Distant Past: The cag Pathogenicity Island of Helicobacter pylori Nicola Pacchiani, Stefano Censini, Ludovico Buti, et al.

RNA-Mediated Regulation in Pathogenic Bacteria Isabelle Caldelari, Yanjie Chao, Pascale Romby, et al.

The Pneumococcus: Epidemiology, Microbiology, and Pathogenesis

Birgitta Henriques-Normark and Elaine I.

Tuomanen

Pathogenesis of Meningococcemia Mathieu Coureuil, Olivier Join-Lambert, Hervé Lécuyer, et al.

Chlamydial Intracellular Survival Strategies Robert J. Bastidas, Cherilyn A. Elwell, Joanne N. Engel, et al.
Mechanisms and Biological Roles of

Contact-Dependent Growth Inhibition Systems

Christopher S. Hayes, Sanna Koskiniemi, Zachary

C. Ruhe, et al.

A Genome-Wide Perspective of Human Diversity and Its Implications in Infectious Disease Jérémy Manry and Lluis Quintana-Murci

Host Specificity of Bacterial Pathogens Andreas Bäumler and Ferric C. Fang

The Inside Story of Shigella Invasion of Intestinal Epithelial Cells

Nathalie Carayol and Guy Tran Van Nhieu

Bartonella and Brucella--Weapons and Strategies for Stealth Attack

Houchaima Ben-Tekaya, Jean-Pierre Gorvel and Christoph Dehio

Concepts and Mechanisms: Crossing Host

Barriers

Kelly S. Doran, Anirban Banerjee, Olivier Disson, et al.

Genome Dynamics in Legionella: The Basis of

Versatility and Adaptation to Intracellular

Replication

Laura Gomez-Valero and Carmen Buchrieser

Mechanisms of Francisella tularensis Intracellular

Pathogenesis

Jean Celli and Thomas C. Zahrt

For additional articles in this collection, see http://perspectivesinmedicine.cshlp.org/cgi/collection/ 